\title{
Using jet breaks to estimate GRB distances
}

\author{
P. Ward, E. J. A. Meurs and C. del Burgo
}

Dunsink Observatory, Castleknock, Dublin 15, Ireland

\begin{abstract}
Recent observations have suggested that the true energy release of GRBs is potentially far less than previously thought. This is due to beaming, a signature of which is a broadband break in the power-law decay of the afterglow emission. Taking these results we have constructed a basic distance estimator, which may be useful as a diagnostic tool for the large amount of GRBs without a spectroscopically measured redshift.
\end{abstract}

Keywords. gamma rays: bursts, cosmology: distance scale.

The value for the isotropic equivalent energy output of a GRB, $E_{i s o}$, in a given bandpass is found by $E_{i s o}=S_{\gamma} \frac{4 \pi D_{l}^{2}}{(1+z)} k$ where $S_{\gamma}$ is the fluence received in the observed band pass and $D_{l}$ is the luminosity distance at redshift $z$. The quantity $k$ is the k-correction. However, it has been shown that true energy release, $E_{\gamma}$, of a GRB is in fact much less than this when the burst is beamed into a collimated jet of half opening angle $\theta_{j}$. $E_{\gamma}$ will therefore be less than $E_{i s o}$ by a factor $\left(1-\cos \theta_{j}\right)$. The beaming fraction can also be described as the ratio of the true energy release and isotropic equivalent energy; $\frac{E_{\gamma}}{E_{i s o}} \approx \frac{\theta_{j}^{2}}{2}$.

One signature of such a jet is a broadband break in the power-law decay of the afterglow emission which occurs at a time $t_{j}$ when the bulk Lorentz factor of the blast wave $(\Gamma)$ has slowed down to $\Gamma<\theta_{j}^{-1}$. According to the formulation made by Sari et al. (1999) the spherical adiabatic evolution of the Lorentz factor is $\gamma(t) \approx 6\left(\frac{E_{i s o}}{n_{1}}\right)^{1 / 8} t_{j}^{-3 / 8}$. Subsequently if the break occurs when $\gamma \approx \theta_{j}^{-1}$, we find that $\left(\frac{2 E_{\gamma}}{E_{\text {iso }}}\right)^{-1 / 2} \approx 6\left(\frac{E_{i s o}}{n_{1}}\right)^{1 / 8} t_{j}^{-3 / 8}$ which can be rearranged to give $E_{i s o}=119\left(2 E_{\gamma}\right)^{4 / 3}(n)^{-1 / 3}\left(t_{j}\right)^{-1}$, giving us an alternative approach for determining $E_{i s o}$.

If GRBs are in fact standard candles the value for $E_{\gamma}$ is a constant. Using this presumption we can use the two equations for $E_{i s o}$ to construct the following relationship between intrinsic burst parameters and the redshift,

$$
\mathbf{D}_{\mathbf{T}}(\mathbf{z})=\mathbf{Q}_{\mathbf{T}}\left(\mathbf{t}_{\mathbf{j}}, \mathbf{S}_{\gamma}, \mathbf{n}, \mathbf{k}\right) \mathbf{E}_{\gamma}^{\mathbf{4} / \mathbf{3}}
$$

We can separate the relationship into a distance quantity $D_{T}$, which is a function of redshift z, and a burst quantity $Q_{T}$, which is a function of the burst properties $t_{j}, S_{\gamma}, n$ and $k$; we define $Q_{T}$ and $D_{T}$ as $Q_{T} \equiv \frac{238.7}{t_{j} n^{(1 / 3)} S_{\gamma} k}$ and $D_{T} \equiv\left[\frac{2 c}{H_{0}}(1+z-\sqrt{(1+z)})\right]^{2} \times$ $\frac{1}{1+z} p c$.

We can now test this relationship using existing data for 12 bursts which have well established values for $z, n, t_{j}, S_{\gamma}$ and $k$. Subsequently, for each burst, we derive a value for $Q_{T}$ (Table 1). Figure 1 shows a plot of $Q_{T}$ vs. z (logarithmic scale). The dotted line represents the observed trend; the value for $Q_{T}$ appears to increase with redshift. Amati et al. (2002) previously noticed a trend of $E_{\text {iso }}$ to increase with z.

The derived relationship is $\mathbf{Q}_{\mathbf{T}}=\mathbf{A} \times \mathbf{z}^{\beta}$, where $\mathrm{A} \sim 0.1 \mathrm{~cm}^{3} \mathrm{erg}^{-1} \mathrm{~s}^{-1}$ and $\beta=1.5$ (see Figure 1). We therefore have a method for determining $z^{*}$, the estimated redshift, according to the relationship derived from the plot in Figure 1: $\mathbf{z} *=\left(\frac{\mathbf{Q}_{\mathbf{T}}}{\mathbf{0 . 1}}\right)^{\mathbf{2} / \mathbf{3}}$. 
Table 1. The parameters used for the 12 bursts in our sample

\begin{tabular}{lcccccc}
\hline GRB & $\mathrm{z}$ & $\mathrm{n}[\mathrm{cm}-3]$ & $t_{j}[\mathrm{days}]$ & $S_{\gamma}\left[10^{-6} \mathrm{erg} \mathrm{cm}-2\right]$ & $\mathrm{k}$ & $Q_{T}\left[10 \mathrm{~cm}{ }^{3} \mathrm{erg}-\mathrm{s}_{\mathrm{s}}^{-1}\right]$ \\
\hline GRB970508 & $0.8349 \pm 0.0003$ & $1 \pm 0.5$ & $25 \pm 5$ & $1.8 \pm 0.3$ & $1.55 \pm 0.08$ & $0.3420 \pm 0.3140$ \\
GRB980329 & $2.95 \pm 0.95$ & $29 \pm 10$ & $0.29 \pm 0.2$ & $65 \pm 5$ & $0.97 \pm 0.09$ & $0.4250 \pm 0.5120$ \\
GRB980703 & $0.9662 \pm 0.0002$ & $28 \pm 10$ & $3.4 \pm 0.5$ & $22.6 \pm 2.26$ & $0.94 \pm 0.08$ & $0.1090 \pm 0.0751$ \\
GRB990510 & $1.6187 \pm 0.0015$ & $0.29 \pm 0.15$ & $1.57 \pm 0.03$ & $19 \pm 2$ & $1.29 \pm 0.03$ & $0.9370 \pm 0.6548$ \\
GRB991208 & $0.7055 \pm 0.0$ & $18 \pm 22$ & $<2.1$ & $100 \pm 10$ & $1.09 \pm 0.03$ & $0.0398 \pm 0.0537$ \\
GRB991216 & $1.02 \pm 0.02$ & $4.7 \pm 6.8$ & $1.2 \pm 0.4$ & $194 \pm 19.4$ & $0.88 \pm 0.09$ & $0.0696 \pm 0.1379$ \\
GRB000301 & $2.0335 \pm 0.0003$ & $26 \pm 12$ & $7.3 \pm 0.5$ & $2 \pm 0.6$ & $1.37 \pm 0.36$ & $0.4028 \pm 0.4402$ \\
GRB000418 & $1.1182 \pm 0.0001$ & $27 \pm 256$ & $25.7 \pm 5.1$ & $20.00 \pm 2$ & $1.00 \pm 0.02$ & $0.0155 \pm 0.1519$ \\
GRB000926 & $2.0369 \pm 0.0007$ & $27 \pm 3$ & $1.8 \pm 0.1$ & $6.20 \pm 0.62$ & $3.91 \pm 1.33$ & $0.1823 \pm 0.0548$ \\
GRB010222 & 1.4769 & $1.7 \pm 0.85$ & $0.93 \pm 0.15$ & $120.00 \pm 3$ & $1.03 \pm 0.04$ & $0.1740 \pm 0.1261$ \\
GRB021004 & 2.3351 & $30 \pm 270$ & $6.5 \pm 0.2$ & $2.55 \pm 0.69$ & $1.04 \pm 0.06$ & $0.4456 \pm 4.1707$ \\
GRB030329 & 0.1685 & $5.5 \pm 2.75$ & $0.48 \pm 0.03$ & $163.00 \pm 1.4$ & $1.01 \pm 0.03$ & $0.1711 \pm 0.1028$ \\
\hline
\end{tabular}
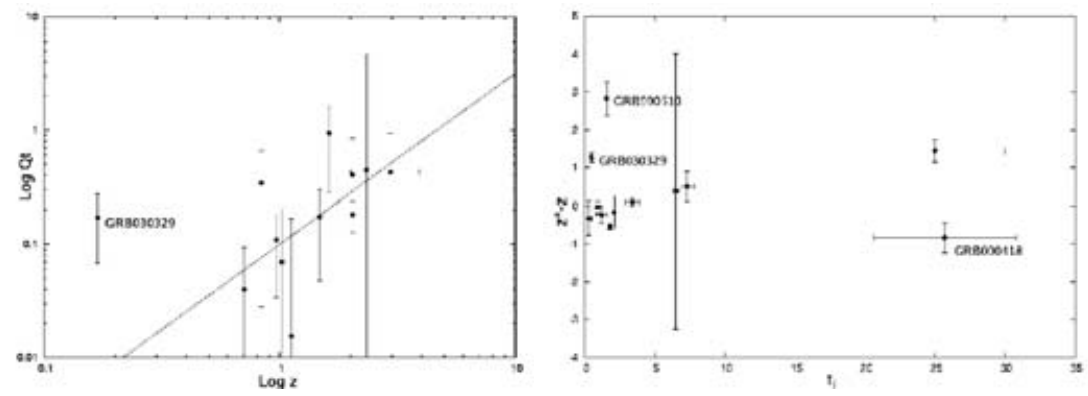

Figure 1. (left) A plot of $\log Q_{T}$ vs. $\log z$. We observe one main outlier to the relationship, GRB030329. This is the only GRB in our sample associated with a supernova, it has the highest fluence and is also the closest at $\mathrm{z}=0.1685$. (right) The dispersion of $t_{j}$ around $\mathrm{z}^{*}$ - $\mathrm{z}$.

If the observed trends are indeed due to some intrinsic characteristic of the bursts, then the above method would be extremely useful not only as a redshift estimator but would also be a useful tool to fill in the many gaps in the current set of GRB results. It is clear however that more data is required to understand fully both the nature of GRBs and their potential as probes of the high redshift universe. Continued research should include data mining of bursts with well established values for $S_{\gamma}, \mathrm{n}, t_{j}$, and z. This would better provide a costraint to the relationship found from Figure 1. Also a more complete treatment involving $D_{T}$ is necessary. If this relationship is true then a plot of $D_{T}$ vs. $Q_{T}$ should give a linear relationship with a slope equal to the value for $E_{\gamma}^{4 / 3}$.

\section{References}

Amati, et al. 2002, A\&A 390, 81

Berger, Kulkarni and Frail 2003, ApJ 590, 379

Bloom, et al. 2001, AJ 121, 2879

Bloom, et al. 2003, ApJ 594, 674

Frail, D. A., Waxman, E., and Kulkarni, S. R. 2000, ApJ 537, 191

Frail, D. A. et al. 2001, ApJ 562, L55

Ghirlanda, et al. 2004, ApJ 616, 331

Rees and Mezsaros 1994, ApJ 430, 93

Rhoads, J. E. 1999, ApJ 525, 737

Sari and Piran 1999, $A \& A$ 138, 537

Sari, R. 1999, ApJ 524, L43

Sari, R., Piran, T., and Halpern, J. P. 1999, ApJ 519, L17

Wei and Lu 1997, A\&A 323, 312 